\title{
Improving ART Pregnancy Rate with Two Kinds of Media and Two Types of Incubators
}

\author{
Bin Wu, Jinzhou Qin, Suzhen Lu, Linda Wu and \\ Timothy J. Gelety
}

Additional information is available at the end of the chapter

http://dx.doi.org/10.5772/intechopen.70062

\begin{abstract}
Culture media and incubators have played a key role in embryo quality. Here, we observed individual patient's embryos to have different response for media and incubators. Patient's 1850 zygotes were divided into two groups randomly and were cultured in Global and in P1 medium. The cleavage rate and embryo quality were recorded. The result showed that the cleavage, top quality embryos on Day 2 and Day 3 were not statistically different between media. However, $45 \%$ patient's embryos grew very well in both Global and P1. 22\% patient's embryos grew well only in Global but poor quality in P1, while $21 \%$ grew well in the Global but poorly in the P1. Only $12 \%$ patient embryos did not grow well in both. The pregnant rate was only $40 \%$ in P1 or $42.5 \%$ in Global ( $P>0.05)$. However, when two media were used simultaneously, the pregnant rate increased to $70.1 \%$. Also, two incubators showed significant higher pregnant rate than in single incubator $(73.2 \%$ vs. $60 \%, \mathrm{P}<0.05)$. In conclusion, the favorable response of individual patient's embryos to media and incubators suggests that using two media and two incubators for embryo culture could significantly improve embryo quality and pregnant rates.
\end{abstract}

Keywords: medium, incubator, IVF, pregnancy, outcome

\section{Introduction}

Assisted reproductive technology (ART) including in vitro fertilization (IVF) and intracytoplasmic sperm injection (ICSI) has now widely been used for the treatment of infertility. The successful application of this technology to human ART is mainly due to embryo 
culture environment innovation including culture media and incubators. So far, different culture systems have been successfully used for in vitro production of human and animal embryos.

Since the first rabbit embryo culture was described in 1912 [1, 2], mouse zygote could be cultured to form blastocyst stage embryos in a complex culture medium [3]. In 1985, an embryo culture medium called human tubal fluid medium (HTF) was first described as designed specifically for human IVF [4]. Since the development of HTF, many modifications and advancements have been made in the recipes for human embryo culture medium.

For many decades, optimization of culture media for the support of human and animal embryos has been a focus of considerable interest [5]. As the further understanding of both the physiological changes in oviduct and uterus and the different metabolic needs of cleavage and blastocyst stage embryos, the many novel embryo culture media are continually developed. Currently, several types of media are available in the market representing different strategies and generally fall into one of the three types: (1) simple salt solutions with added energy substrates, such as KSOM, P1, etc., [6, 7]; (2) complex tissue culture media, such as Ham's F-10 [8]; and (3) sequential media, such as G1/G2, and developed G5 serious media [9]. More recently, sequential culture media have been produced to take into account the changing metabolic needs of the embryo from the cleavage to the blastocyst stage [10,11]. So far, many commercial embryo culture media are available for human embryo culture and their effect for embryo culture is various [12]. At present, when we search with key words "human embryo culture medium comparison" in google.com, the 53,600 results will occur at $0.6 \mathrm{~s}$. The studies comparing these medium effects on embryonic development have reported contradictory conclusion. Many studies did not find a significant difference or just tiny difference between various culture media [13, 14]. Recently, Mantikou et al. [15] used meta-analysis to evaluate 31 different comparisons for 20 different culture media and did not find what culture medium leads to the best success rates in IVF/ICSI.

We think that significant differences of various media for embryo culture may be difficult to be demonstrated because every commercial company for human embryo culture media must continuously improve the quality of its available culture media. Thus, most of the current commercial culture media may produce satisfied results for human embryo culture. Therefore, the choice of the best culture medium in each laboratory has been attributed to embryologist interesting and specific work conditions.

Also, incubators in the IVF laboratory play a pivotal role in providing a stable and appropriate culture environment required for optimizing embryo development and clinical outcomes. With technological advances, several types of incubators have been applied to human IVF laboratory. Recently, Swain [16] did a comparative analysis of embryo cultural incubators in human IVF laboratories and reviewed some incubator functions and key environmental variables controlled and the technology utilized in various units. This comparison indicates that smaller bench top/top load incubators provide faster recovery of environmental variables, but there is no clear advantage of any particular incubator based on clinical outcomes. 
Based on the last decade practice of our IVF center, there was no any difference on embryo culture between Cook Minc incubator and front-door big-box incubator. However, we observed that the same patient's sibling embryos for splitting into two medium cultures often have different development results under the condition of different incubators. Our question is whether patient's embryos have a favorable selection for culture medium or incubator condition? The objective of this study is to determine whether specific differences of patient embryos in response to culture media and incubator are important in the human embryo culture system.

\section{Materials and methods}

\subsection{Culture media}

This study mainly used two media for embryo culture: P1 medium is from Irvine Scientific, Inc, CA, and Life-Global medium is from Life Global, LLC, Canada. Two kinds of media added 10\% serum substitute supplement (SSS) (Irvine Scientific, Inc, CA) for embryo culture.

\subsection{Culture incubators}

Forma water-jacketed $\mathrm{CO}_{2}$ incubator is from Thermal Forma Scientific, Inc, and this incubator is just connected to medical grade $\mathrm{CO}_{2}$ gas tank and is adjusted to $5 \% \mathrm{CO}_{2}$ for embryo culture. Cook Benchtop Incubator was connected to the certified premixed tri-gas tank which contained $5 \% \mathrm{O}_{2}, 6 \% \mathrm{CO}_{2^{\prime}}$ and $89 \% \mathrm{~N}$ (Figure 1). Although two incubators connected with different $\mathrm{CO}_{2}$ concentration, their $\mathrm{pH}$ tests showed the range from 7.21 to 7.38 and no significant difference was observed between two medium in two types of incubators.

\section{Embryo Culture Incubator}
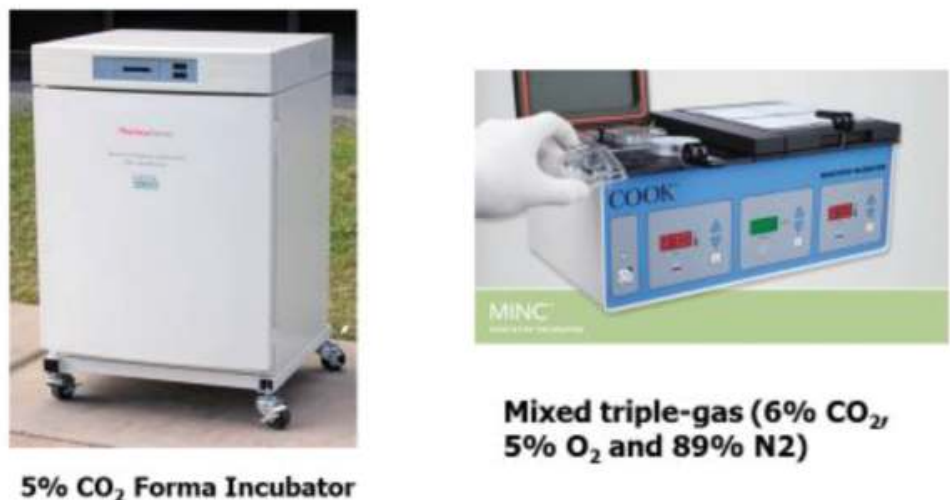

Mixed triple-gas $\left(6 \% \mathrm{CO}_{2}\right.$ $5 \% \mathrm{O}_{2}$ and $89 \% \mathrm{N2}$ )

Figure 1. Embryo culture incubators. Two kinds of incubators are shown as big-box incubator (left) and Cook Bench Minc incubator (right). 


\section{Experiment design}

This was a prospective randomized study of infertility couples undergoing assisted reproductive procedures during 2012-2013 at the Arizona Center for Reproductive Endocrinology and Infertility. During this period, all patients (age 25-43) were treated by our standard stimulation protocol (LA/HMG, HCG 10,000 IU/ml) before oocyte retrieval. The retrieval oocytes were cultured in P1 medium (Irvine Scientific, Inc) with 3\% human serum albumin (HSA, InVitoCare, Frederick, MD) for $4-6 \mathrm{~h}$ at $37^{\circ} \mathrm{C}$ with $5 \% \mathrm{CO}_{2}$ incubator. Then, oocytes were routinely inseminated in $100 \mu \mathrm{l}$ of P1 medium microdrops for in vitro fertilization or conducted by intracytoplasmic sperm injection depending on husband sperm quality (approximately $35 \%$ of cycles required ICSI). Fertilization was assessed on the following day, 18-20 $\mathrm{h}$ after insemination or ICSI. If two distinct pronuclei (2PN) were observed, the fertilization was confirmed. The zygotes per patient were randomly divided into two groups and cultured in either P1 medium or Life-Global medium in petri dishes with each drop of $50 \mu 1$ culture medium for another 2 days (see experimental design, Figure 2).

Each embryo was cultured in an individual microdrop. The status of embryo cleavage and quality were assessed after a further 24 and $48 \mathrm{~h}$ of in vitro culture. The embryonic grade was evaluated according to the number and size equality of blastomeres, presence or absence of granularity, and the relative proportion of anucleate fragments by at least two experienced embryologists by 100X magnification on an inverted microscope. Based on our standard criteria, good-quality or top-quality embryos (Grade 5) were defined as regular, spherical blastomeres with less 10\% extracellular fragmentations and had 6-10 blastomeres on day 3 . Embryos with Grade 1-4 were defined as low quality. On the day of embryo transfer (ET), one

\section{Embryo Culture Method}

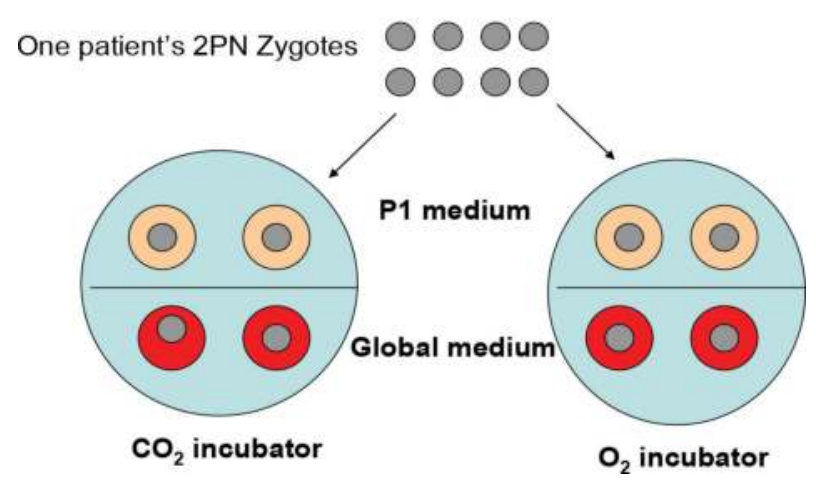

Figure 2. Embryo culture method. A patient sibling fertilized zygotes were randomly divided into two media and two incubators for culture. 
to four embryos were selected for abdominal ultrasound-guided transfer to uterus according to patient age and embryo quality. The transferred embryos from each medium or incubator were recorded. The most of, most of the best quality embryos for transfer were selected from two media and two incubators. The remaining embryos, if any, were left in the culture dish to undergo cryopreservation. Clinical pregnancy was diagnosed by the presence of a gestational sac by ultrasound echographic screening approximately 5-6 weeks after the embryo replacement procedure.

Statistical analysis was performed using student $t$-test. Significant statistical difference was considered as $P<0.05$.

\section{Results}

\subsection{New discovery}

In 2008-2009, we often observed that the patient's embryos had a different response to each medium. Some patient's embryos favored to grow in the global medium, while some patient's embryos preferred to live in the P1 medium and some embryos grew very well in both global medium and P1 medium, which means that embryos have a favorable selectivity to medium (Figure 3).

\section{The same day embryos for two patients}

\section{P1}

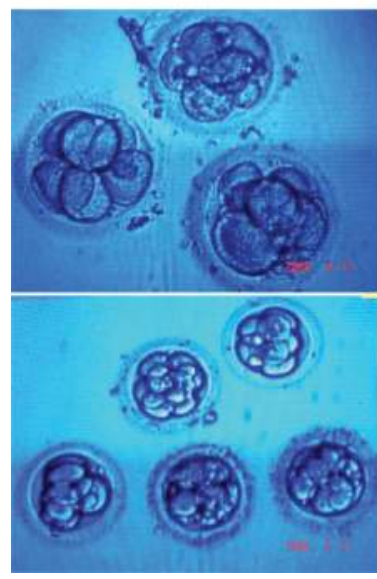

Global

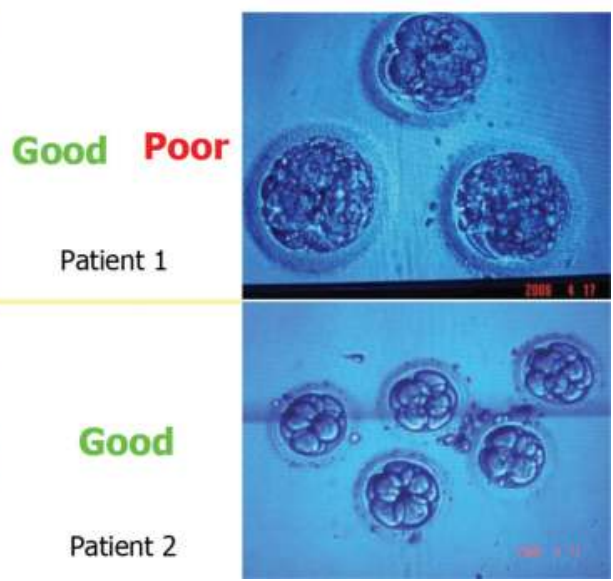

Figure 3. The patient's embryo response to two media. On the same day, two patients' embryos were placed in the two medium culture. Patient 1 embryos grew very well in the P1 medium (left), but they had a lot of fragmentation in global medium (right). However, the 10 embryos of patient 2 grew very well in both media. 


\subsection{Experimental verification}

In order to verify our former observation, two different commercially available media (P1 and Life-Global media) were used in this study. A total of 1850 normal fertilized 2PN zygotes from 220 consecutive patient cycles were studied. The cleavage rate and top-quality embryos on day 2 and day 3 were compared. Results indicated the same zygote cleavage rate for two media, and global medium seemed to yield slightly higher top-quality embryos on day 2 and day 3, but it was not statistically different between two media ( $P>0.05$, Table 1 and Figure 4). When patient's sibling embryos were cultured in two different incubators, their cleavage and the top embryo of day 2 and day 3 also did not show any significant difference $(P>0.05$, Figure 5).

However, when the patient sibling embryos were cultured in two media, some patient embryos developed very well in the P1 medium, while some patient embryos grew well in global medium. Here, we gave four patient samples to show the responses of patient embryos to two culture media (Figures 6-9).

Patient A was 38 years old. Five oocytes were retrieved on September 30, 2013, and four zygotes were individually cultured in P1 and global medium in Forma incubator and Cook Minc incubator, respectively. On day 3, all embryos showed a good quality under the various conditions

Patient B was 23 years old. Thirteen oocytes were retrieved on October 4, 2013, and four zygotes were individually cultured in P1 and global medium in Forma incubator and Cook Minc incubator, respectively. On day 3, all embryos showed a low quality and slow growing under the various conditions.

Patient C was 33 years old. Fourteen oocytes were retrieved on September 30, 2013, and 11 zygotes were cultured in P1 and global microdrop medium (one embryo in each drop) in

\begin{tabular}{|c|c|c|c|c|c|c|c|c|}
\hline \multirow{3}{*}{$\begin{array}{l}\text { Embryo } \\
\text { grade }\end{array}$} & \multicolumn{4}{|l|}{ Medium } & \multicolumn{4}{|l|}{ Incubator } \\
\hline & \multicolumn{2}{|l|}{ Global } & \multicolumn{2}{|l|}{ P1 } & \multicolumn{2}{|l|}{ Forma } & \multicolumn{2}{|l|}{ Minc } \\
\hline & $\begin{array}{l}\text { Embryos/ } \\
\text { total }\end{array}$ & $\%$ & $\begin{array}{l}\text { Embryos/ } \\
\text { total }\end{array}$ & $\%$ & $\begin{array}{l}\text { Embryos/ } \\
\text { total }\end{array}$ & $\%$ & $\begin{array}{l}\text { Embryos/ } \\
\text { total }\end{array}$ & $\%$ \\
\hline Cleavage & $907 / 930$ & $97.5^{\mathrm{a}}$ & $808 / 920$ & $98.7^{\mathrm{a}}$ & $992 / 1025$ & $96.8^{\mathrm{a}}$ & $807 / 825$ & $97.8^{\mathrm{a}}$ \\
\hline $\begin{array}{l}\text { Day } 2 \\
\text { top-quality } \\
\text { embryos }\end{array}$ & $633 / 845$ & $74.9^{\mathrm{a}}$ & $624 / 836$ & $74.6^{\mathrm{a}}$ & $730 / 988$ & $73.9^{\mathrm{a}}$ & $598 / 800$ & $74.8^{\mathrm{a}}$ \\
\hline $\begin{array}{l}\text { Day } 3 \\
\text { top-quality } \\
\text { embryos }\end{array}$ & $564 / 857$ & $65.8^{\mathrm{a}}$ & $477 / 768$ & $62.1^{a}$ & $573 / 902$ & $63.3^{\mathrm{a}}$ & $478 / 726$ & $65.8^{\mathrm{a}}$ \\
\hline
\end{tabular}

${ }^{a}$ No significant difference between two medium groups $(P>0.05)$.

Some patient embryos were not observed on day 2 and were observed on day 3. Day 2 top-quality embryo shows 2-6 cells/Grade 5 and day 3 top-quality embryo shows 5-8 cells/Grade 5.

Table 1. Effect of culture media and incubators on zygote development. 


\section{Global and P1 medium comparison}

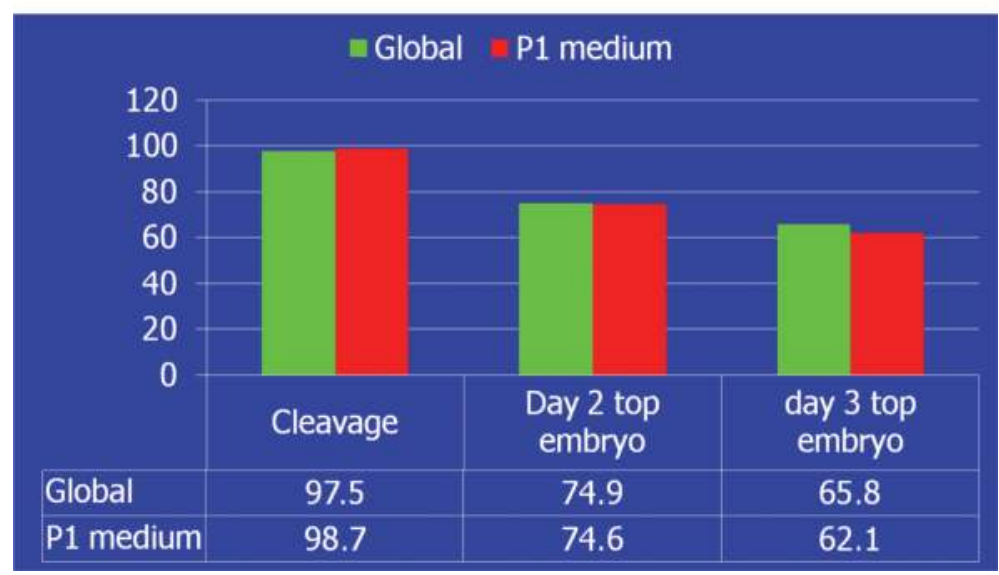

Figure 4. Patient embryos cultured in two medium did not show any significant difference on cleavage, day 2 and day 3 high-quality embryos.

\section{Forma and Minc incubator comparison}

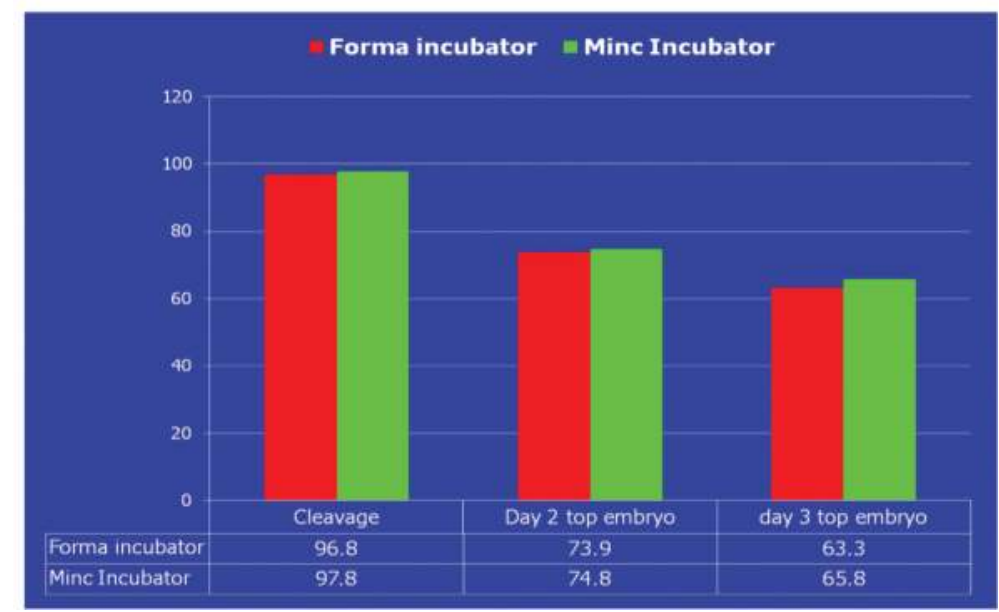

Figure 5. Patient embryos cultured in two different incubators did not show any significant difference on cleavage, day 2 and day 3 high-quality embryos.

Forma incubator and Cook Minc incubator, respectively. On day 3, only three good-quality embryos were obtained in the global medium in the Cook Minc incubator. Other eight embryos showed low quality under other three kinds of condition. 
Patient A (38 yrs old, 5 oocytes on 10/3/2013)

\section{Four embryos in P1 and Global media in two incubators}

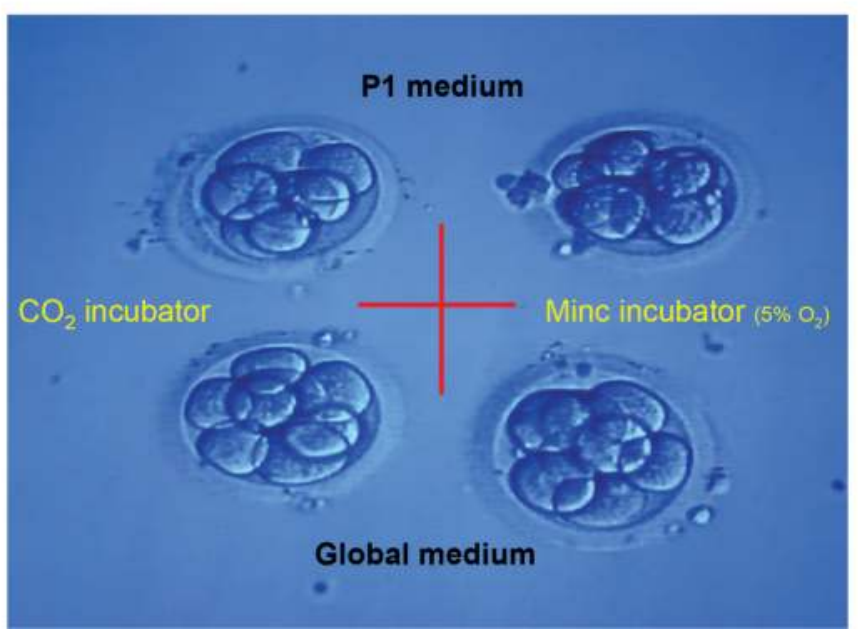

Figure 6. The four embryos of a 38-year-old woman showed very well growing in two media and two incubators. Day 3 embryos were displayed under the same microscope view.

\section{Patient B (23 yrs old, 13 oocytes on 10/7/2013) Day 3 embryos in P1 and Global media in two incubators}

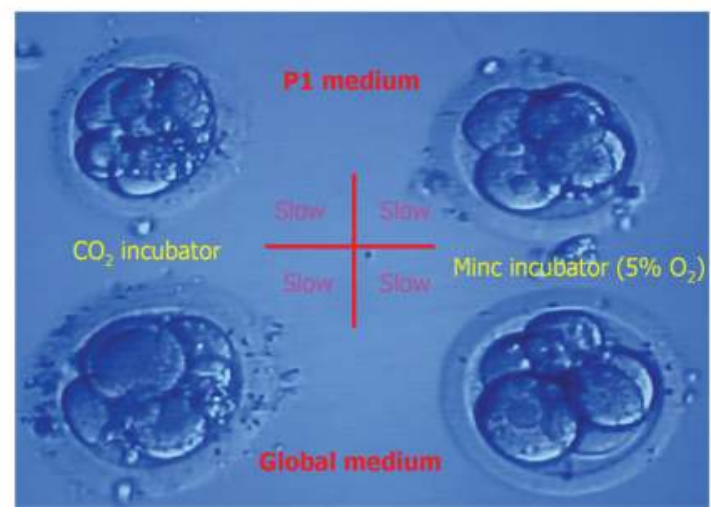

Figure 7. The four embryos of a 23-year-old woman showed very poor growing in two media and two incubators. Day 3 embryos were displayed under the same microscope view.

Patient D was 41 years old. Five oocytes were retrieved on October 4, 2013, and five zygotes were individually cultured in P1 and global medium in Forma incubator and Cook Minc incubator, respectively. On day 3, only one embryo in P1 medium with Forma incubator showed poor quality and other four embryos had good quality. 
Patient C (33 yrs old, 14 oocytes on 10/3/2013)

11 embryos in P1 and Global media in two incubators

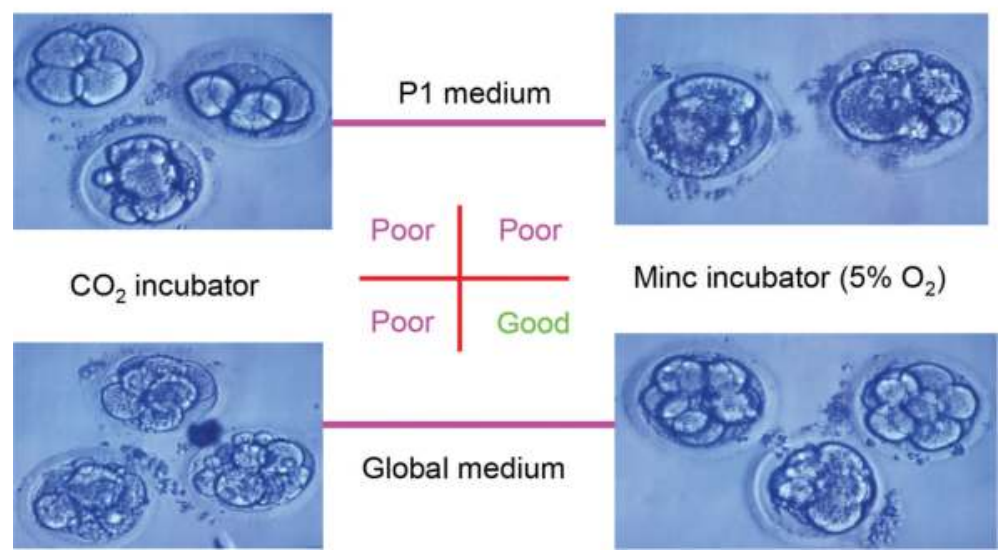

Figure 8. The eight embryos of a 33-year-old woman showed very poor growing with P1 medium in two incubators and global medium in big-box incubator. However, three good-quality embryos were obtained in the global medium with Minc incubator.

\section{Patient D (41 yrs old, 5 oocytes on 10-7-2013)}

\section{Five embryos in P1 and Global media in two incubators}
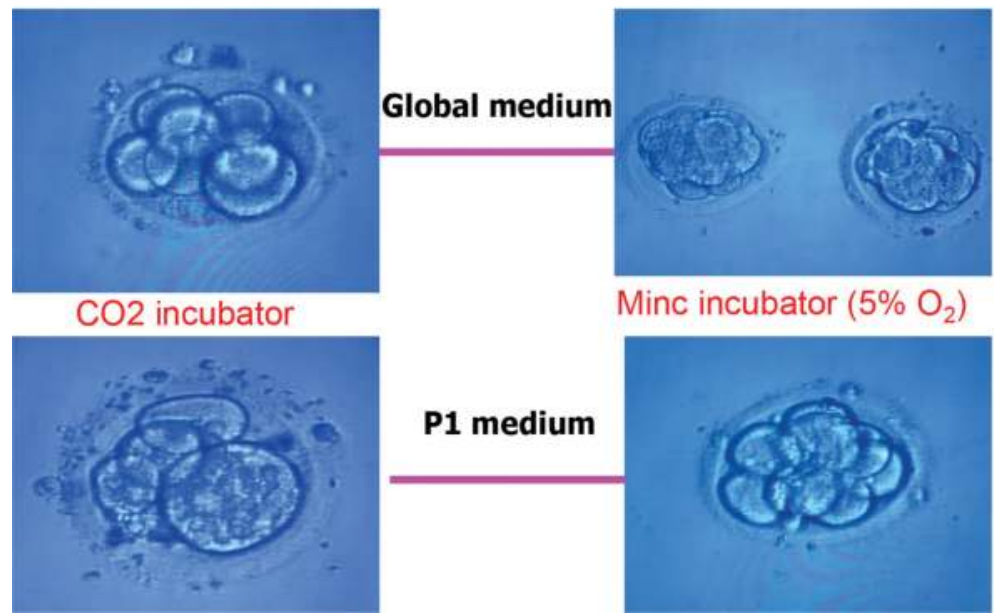

Figure 9. The four embryos of a 41-year-old woman showed very good growing with global medium in two incubators and Pl medium in the Cook Minc incubator. Only one embryo was of poor quality in the P1 medium with big-box incubator. 
These results showed that the different patient's embryos had different responses to media. In order to compare large number of data, 1875 embryos of 174 patients were divided into four groups. The first group contains $45 \%$ (78/174) patient's embryos growing very well in either global medium or P1 medium. The second group contains 22\% (38/174) patient's embryos growing well only in global medium with poor quality in P1 medium. The third group contains $21 \%$ (37/174) growing well in the P1 medium but poorly in the global medium and the fourth group contains $12 \%$ (21/174) not good growing in both P1 and global media (Table 2). In order to clearly show the data of table, a bar graph was drawn (Figure 10). We may very clearly see some patient embryos growing very well in global medium or P1 medium, which showed patient embryo selectivity.

\begin{tabular}{|c|c|c|c|c|c|}
\hline \multirow{2}{*}{$\begin{array}{l}\text { Embryo quality in } \\
\text { medium }\end{array}$} & \multirow{2}{*}{$\begin{array}{l}\text { Patients/total } \\
\text { patients }(\%)\end{array}$} & \multicolumn{2}{|l|}{ Global medium } & \multicolumn{2}{|l|}{ P1 medium } \\
\hline & & Embryo number & $\begin{array}{l}\text { Top quality } \\
\text { mean } \pm S D\end{array}$ & Embryo number & $\begin{array}{l}\text { Top quality } \\
\text { mean } \pm S D\end{array}$ \\
\hline $\begin{array}{l}\text { Good in global } \\
\text { and P1 }\end{array}$ & $78 / 174(45 \%)$ & $391 / 448$ & $87.6 \pm 16.3^{\mathrm{a}}$ & $359 / 425$ & $84.5 \pm 16.2^{\mathrm{a}}$ \\
\hline $\begin{array}{l}\text { Good in global but } \\
\text { poor in P1 }\end{array}$ & $38 / 174(22 \%)$ & $153 / 190$ & $80.7 \pm 22.8^{\mathrm{a}}$ & $67 / 205$ & $32.8 \pm 19.1^{\mathrm{b}}$ \\
\hline $\begin{array}{l}\text { Good in P1 but } \\
\text { poor in global }\end{array}$ & $37 / 174(21 \%)$ & $51 / 185$ & $27.7 \pm 20.6^{\mathrm{a}}$ & $137 / 200$ & $68.3 \pm 22.5^{\mathrm{b}}$ \\
\hline $\begin{array}{l}\text { Poor in global } \\
\text { and P1 }\end{array}$ & $21 / 174(12 \%)$ & $30 / 105$ & $28.8 \pm 17.7^{\mathrm{a}}$ & $26 / 120$ & $21.9 \pm 20.9^{\mathrm{a}}$ \\
\hline
\end{tabular}

Note: The same superscript in each row indicates no significant difference $(P>0.05)$, and different superscript in each row indicates significant difference $(P<0.05)$.

Table 2. Patient sibling embryos in response to different culture media.

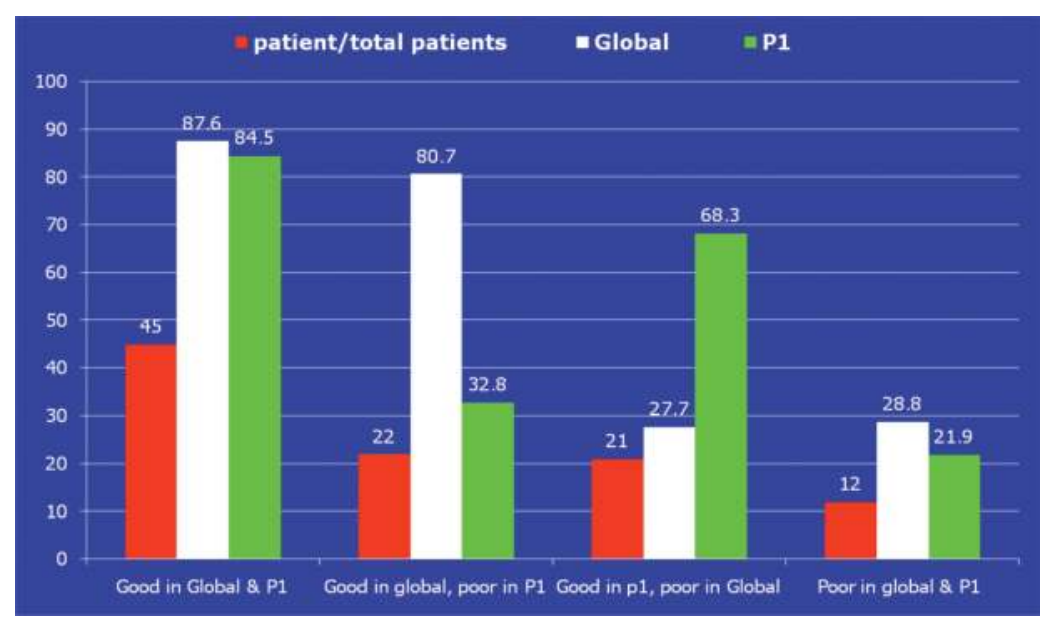

Figure 10. Growing distribution of patients' embryos in two media for culture. The first bar in each group represents percentage of patient's embryos in each group. P1 indicates P1 medium and G indicates global medium. The data indicate percentage. 


\section{Pregnancy rate in two media and two incubators}

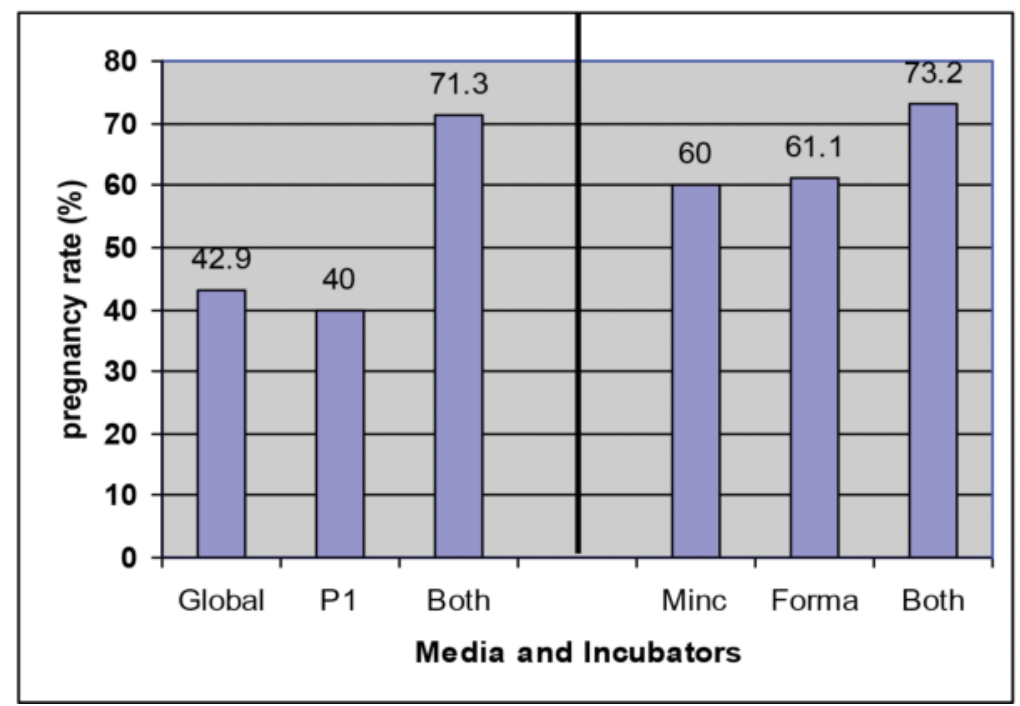

Figure 11. The statistics of patient pregnant rates in two media and two incubators.

The statistics of the pregnant rate showed 40\% (10/25) in P1 medium and 42.5\% (9/21) in global medium $(P>0.05)$. However, when two media were used simultaneously for patient embryo culture, the pregnant rate significantly increased to $70.1 \%(122 / 174)$. At the same time, when two media were cultured in two incubators, it had a significant higher pregnant rate than in single incubator $(73.2 \%$ vs. $60 \%, P<0.05$, Figure 11$)$.

\section{Discussion}

In the last near four decades, assisted reproductive technologies have been widely applied to the treatment for infertile couples to realize their dream to have baby in their family. However, the current successful rate is still kept in low level about $40 \%$. Thus, vast efforts have been undertaken to improve IVF pregnancy rate by continuously improving and modifying in vitro culture medium system and innovating in embryo selection techniques such as timelapse, preimplantation embryo diagnosis and screening (PGD/PGS). So far, numerous studies have been reported on different culture medium formulations and their effects on embryo cleavage and blastocyst formation [12]. Although current commercial culture medium composition varies widely, all of them may support human embryo in vitro culture growing very well. Thus, the selection of embryo culture medium depends on laboratory embryologist's favor and custom. 
However, we are reporting a new observation which showed patient's embryo-specific differences in response to culture media in clinical IVF-ET. Our results indicated that some patient's embryos favored to grow in the global medium, while some patient's embryos preferred to live in the P1 medium and some embryos grow very well in both the global medium and P1 medium; some patient's embryos did not grow well in both P1 and global media, which means that different patient embryos have a favorable selectivity to culture medium. The aim of embryo culture after in vitro fertilization is to obtain good-quality embryos for transfer into women uterus. Because of patient's embryo selectivity, when all embryos of a patient are placed in a single medium culture, it is possible that all embryos are either very good or very poor. If embryos show poor quality in the single medium, this patient may be a failure in this IVF cycle. However, when embryos are cultured in two media, some embryos may be poor quality in a medium, but some embryo may be good quality in another medium. Thus, this patient still has good-quality embryos for transfer in order to make sure an increase in pregnancy opportunity.

Our statistic results showed that $45 \%$ patient's embryos grow very well in either global medium or P1 medium. Thus, the embryos from these $45 \%$ patients always grow very well no matter what media were used in either P1 or global medium. They are also easy to obtain successful pregnancy group. In addition, the embryos of about $12 \%$ patients could not grow well in both P1 and global media. These patients of this group are very difficult to get pregnancy because they cannot get any good-quality embryos for transfer using any medium. This may be due to patient oocyte quality or sperm quality. The embryos of remaining about $43 \%$ patients displayed a real medium selection. That means that $22 \%$ patient's embryos were growing very well only in P1 medium but poor quality in global medium, while the embryos of $21 \%$ patients grew well in the global medium but poorly in the P1 medium. Thus, we may obtain high-quality embryos from this $43 \%$ patient group by the selection of two culture media. In this way, the best estimation of IVF successful rate may reach to $45+43=88 \%$ of patients under the current IVF technology. In our statistics based on various ages of transfer embryo women, the pregnant rate of each group in two media and two types of incubators are listed in Table 3. However, this very high pregnancy rate resulted in $20.7 \%$ twin and $3.74 \%$ triplet baby birth, which showed that two medium cultures really increased transfer embryo implantation opportunity. In clinical practice, the number of transfer embryos should be reduced significantly accordingly.

\begin{tabular}{lll}
\hline Patient age & Transfer embryo no. and range & Pregnant no. and rate (\%) \\
\hline$<28$ & $1.98(1-2)$ & $20 / 23(86.96 \%)$ \\
$28-34$ & $2.64(1-3)$ & $50 / 67(74.63 \%)$ \\
$35-27$ & $2.94(2-4)$ & $24 / 33(72.72 \%)$ \\
$38-40$ & $3.12(1-4)$ & $21 / 32(65.63 \%)$ \\
$>40$ & $3.81(2-4)$ & $9 / 21(42.86 \%)$ \\
\hline
\end{tabular}

Table 3. The result of pregnancy with two media and two types of incubators for embryo culture and mixed embryo transfer. 
Transferring embryos from two media may significantly improve human IVF pregnancy rate. Wirleitner et al. [17] ever reported an interesting observation in which the transfer of two embryos where one embryo was cultured in either medium resulted in a significantly high rate of twin pregnancies. Our research showed that two medium cultures might obtain $71 \%$ pregnancy rate. However, if single medium was used for culture, it may just produce about may produce about $40 \%$ pregnant rates. Using two media in one incubator for culture may increase to $60 \%$ pregnancy rate. When two media plus two incubators were used, the pregnancy might increase to $73 \%$. Thus, the application of two media and two types of incubators may significantly improve human IVF/ICSI clinical pregnancy.

\section{Conclusions}

Patient-specific variability in response to commercially available media appears to play a significant role in clinic IVF practice, and the application of two media and two types of incubators for each patient embryo culture enables to ensure every patient to have sufficient high-quality embryos for transfer. The favorable response of individual patient's embryos to media and incubators suggests that in IVF clinic practice, using two media and two incubators for embryo culture could significantly improve IVF/ICSI pregnant rates.

\section{Author details}

Bin $\mathrm{Wu}^{1 *}$, Jinzhou Qin ${ }^{2}$, Suzhen $\mathrm{Lu}^{1}$, Linda $\mathrm{Wu}^{1}$ and Timothy J. Gelety ${ }^{1}$

*Address all correspondence to: bwu13@yahoo.com

1 Arizona Center for Reproductive Endocrinology and Infertility, Tucson, Arizona, United States

2 Reproduction Medical Center, Luohu Hospital Affiliated to Shenzhen University, Shenzhen, Guangdong, China

\section{References}

[1] Brachetm A. Recherches sur la de"terminisme he're'ditaire de l'oeuf des mammife'res. De'veloppement in vitro de jeunes ve'sicules blastodermiques de lapin. Archives de Biologie (lie'ge). 1913;28:423-426

[2] Biggers JD. IVF and embryo transfer: Historical origin and development. Fertility Magazine. 2013;16:5-15

[3] Whitten WK. Culture of tubal mouse ova. Nature. 1956;177(4498):96

[4] Quinn P, Kerin JF, Warnes GM. Improved pregnancy rate in human in vitro fertilization with the use of a medium based on the composition of human tubal fluid. Fertility and Sterility. 1985;44(4):493-498 
[5] Biggers JD. Thoughts on embryo culture conditions. Fertility Magazine. 2014;16:34-43

[6] Biggers JD. History of KSOM, a single medium for embryo culture. Fertility World. 2005;3:4-7

[7] Rieger D. A single medium can support development of human embryos to the blastocyst stage. Fertility World. 2005;3:24-27

[8] Edwards RG. Test-tube babies, 1981. Nature. 1981;293:253-256

[9] Gardner DK, Lane M. Embryo culture systems. In: Trounsoon AO, Gardner DK, editors. Handbook of In Vitro Fertilization. 2nd ed. CRC Press LLC; The United State of America 2000. pp. 205-264

[10] Fong C-Y, Bongso A. Comparison of human blastulation rates and total cell number in sequential culture media with and without co-culture. Human Reproduction. 1998;14:774-781

[11] Morbeck DE, Baumann NA, Oglesbee D. Composition of single-step media used for human embryo culture. Fertility and Sterility. 2017;107(4):1055-1060. DOI: 10.1016/j. fertnstert.2017.01.007

[12] Morbeck DE, Krisher RL, Herrick JR, Baumann NA, Matern D, Moyer T. Composition of commercial media used for human embryo culture. Fertility and Sterility. 2014;102(3): 759-766

[13] Aoki VW, Wilcox AL, Peterson CM, Parker-Jones K, Hatasaka HH, Huang I, Carrell DT. Comparison of four media types during 3-day human IVF embryo culture. Reproductive BioMedicine Online. 2005;10(5):600-606. DOI: 10.1016/S1472-6483(10)61667-7

[14] Chronopoulou E, Harper JC. IVF culture media: Past, present and future. Human Reproduction Update. 2015;21(1):39-55. DOI: https:/doi.org/10.1093/humupd/dmu040

[15] Mantikou E, Youssef MAFM, van Wely M, van der Veen F, Al-Inany HG, Repping S, Mastenbroek S. Embryo culture media and IVF/ICSI success rates: A systematic review. Human Reproduction Update. 2013;19(3):210-220

[16] Swain JE. Optimal human embryo culture. Seminars in Reproductive Medicine. 2015; 33(2):103-117. DOI: 10.1055/s-0035-1546423

[17] Wirleitner B, Vanderzwalmen P, Stecher A, Zech MH, Zintz M, Zech NH. Individual demands of human embryos on IVF culture medium: Influence on blastocyst development and pregnancy outcome. Reproductive BioMedicine Online. 2010;21:776-782 